\title{
High Resolution Spectroscopy of Neutral Yb Atoms in a Solid Ne Matrix
}

\author{
R. Lambo, ${ }^{1,}$ 网 C.-Y. Xu (徐晨昱), ${ }^{2,3,}$, S. T. Pratt,${ }^{4}$ H. Xu (徐红) ${ }^{4}$ J. C. \\ Zappala, ${ }^{2}$ K. G. Bailey, ${ }^{2}$ Z.-T. Lu (卢征天),${ }^{5}$ P. Mueller, ${ }^{2}$ T. P. O'Connor, ${ }^{2}$ B. B. \\ Kamorzin ${ }^{6}$ D. S. Bezrukov, ${ }^{6,7}$ Y. Xie, ${ }^{1}$ A. A. Buchachenko, ${ }^{6}$, 用 and J. T. Singh ${ }^{2,8}$, 周 \\ ${ }^{1}$ Shenzhen Institutes of Advanced Technology, Chinese Academy of Sciences, Shenzhen, 518055, China \\ ${ }^{2}$ Physics Division, Argonne National Laboratory, Argonne, Illinois 60439, USA \\ ${ }^{3}$ Department of Physics and Enrico Fermi Institute, \\ University of Chicago, Chicago, Illinois 60637, USA \\ ${ }^{4}$ Chemical Science and Engineering Division, Argonne National Laboratory, Lemont, Illinois 60439, USA \\ ${ }^{5}$ Hefei National Laboratory for Physical Sciences at the Microscale, \\ CAS Center for Excellence in Quantum Information and Quantum Physics, \\ University of Science and Technology of China, 96 Jinzhai Road, Hefei 230026, China \\ ${ }^{6}$ CEST, Skolkovo Institute of Science and Technology, \\ Skolkovo Innovation Center, Moscow 121205, Russia \\ ${ }^{7}$ Department of Chemistry, M.V. Lomonosov Moscow State University, Moscow 119991, Russia \\ ${ }^{8}$ National Superconducting Cyclotron Laboratory, Michigan State University, East Lansing, Michigan 48824, USA
}

(Dated: July 12, 2021)

\begin{abstract}
We present an experimental and theoretical study of the absorption and emission spectra of $\mathrm{Yb}$ atoms in a solid Ne matrix at a resolution of $0.025 \mathrm{~nm}$. Five absorption bands were identified as due to transitions from the $4 f^{14} 5 d^{0} 6 s^{2}{ }^{1} S_{0}$ ground state configuration to $4 f^{14} 5 d^{0} 6 s 6 p$ and $4 f^{13} 5 d^{1} 6 s^{2}$ configurations. The two lowest energy bands were assigned to outer-shell transitions to $6 s 6 p{ }^{3} P_{1}$ and ${ }^{1} P_{1}$ atomic states and displayed the structure of a broad doublet and an asymmetric triplet, respectively. The remaining three higher-frequency bands were assigned to inner-shell transitions to distinct $J=1$ states arising from the $4 f^{13} 5 d^{1} 6 s^{2}$ configuration and were highly structured with narrow linewidths. A classical simulation was performed to identify the stability and symmetry of possible trapping sites in the Ne crystal. It showed that the overarching $1+2$ structure of the high frequency bands could be predominantly ascribed to crystal field splitting in the axial field of a 10atom vacancy of $C_{4 v}$ symmetry. Their prominent substructures were shown to be manifestations of phonon sidebands associated with the zero-phonon lines on each crystal field state. Unprecedented for a metal-rare gas system, resolution of individual phonon states on an allowed electronic transition was possible under excitation spectroscopy which reflects the semi-quantum nature of solid Ne. In contrast to the absorption spectra, emission spectra produced by steady-state excitation into the ${ }^{1} P_{1}$ absorption band consisted of simple, unstructured fluorescence bands.
\end{abstract}

PACS numbers:

\section{INTRODUCTION}

Rare gas (RG) solids, formed at cryogenic temperatures, are a promising host medium for the capture, detection and quantum state manipulation of guest atoms and molecules. They provide stable and chemically inert isolation and confinement for a wide variety of guest species at a tunable density - from a single isolated atom to a number possibly exceeding $\sim 10^{16}$ atoms $/ \mathrm{cm}^{3}$. Because RG solids are transparent at optical wavelengths, the guest species can be probed using lasers and the induced fluorescence efficiently detected outside the solid. Spin coherence times of the guest species, which are ultimately dominated by long-range dipolar couplings [1, 2, could be made as long as $10^{3} \mathrm{~s}$ for nuclear spins and $1 \mathrm{~s}$ for electronic spins by minimizing spin impurities within

*These two authors contributed equally.

†Electronic address: a.buchachenko@skoltech.ru

${ }^{\ddagger}$ Electronic address: singhj@frib.msu.edu the host matrix. Applications of this "matrix isolation" technique include tests of fundamental symmetries [3, 4, magnetometry [5, 6] and quantum information science 7].

Examples of systems studied specifically for these applications include alkali atoms in both solid RGs 8 and parahydrogen [9, Yb atoms in solid Ne 10, 11] and Tm atoms in solid $\mathrm{Ar}$ and $\mathrm{Kr}$ 12. For these species, medium effects generally broaden linewidths and shift transition frequencies by several hundred wavenumbers $[8]$. Despite substantial matrix-induced perturbations to their $D$-lines, however, alkali atoms have been successfully optically pumped in matrix isolation [5, 13. Furthermore, spin coherence times as long as $0.1 \mathrm{~s}$ have been observed for $\mathrm{Rb}$ atoms in solid parahydrogen [6].

Ytterbium $(\mathrm{Yb})$ is a heavy divalent atom with a ${ }^{1} S_{0}$ electronic ground state, several optical transitions and a naturally abundant isotope, ${ }^{171} \mathrm{Yb}(14 \%)$, whose nuclear spin is $1 / 2$. These features combine to make matrixisolated ${ }^{171} \mathrm{Yb}$ a promising candidate for a solid state search for a permanent electric dipole moment [14] or for a nuclear spin-based qubit. Such applications depend 


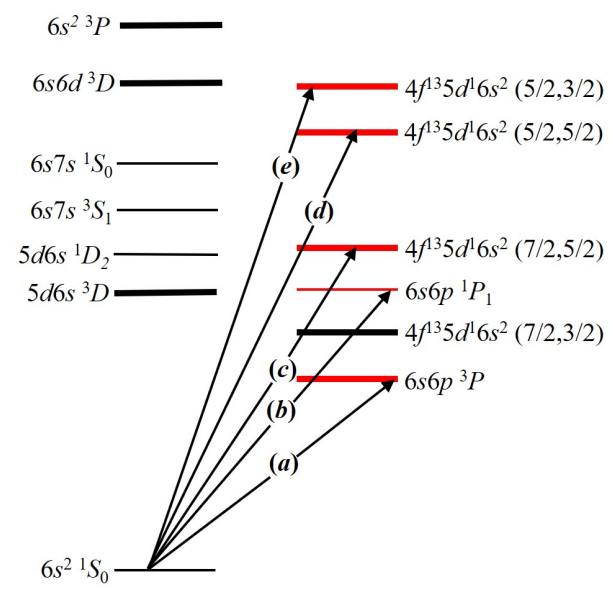

FIG. 1: Low-lying energy levels of the $\mathrm{Yb}$ atom with the fine structures omitted. The arrows represent five transitions observed in solid Ne labelled from (a)-(e) following Tables I and II They correspond to $E 1$ transitions from the $6 s^{2}{ }^{1} S_{0}$ ground state to $J=1$ states in five fine structure manifolds.

on the ability to both optically prepare and readout the ${ }^{171} \mathrm{Yb}$ nuclear spin state. This in turn depends crucially on the strength of the hyperfine interaction in the excited state compared to the size of the matrix-induced perturbations. The need to characterize the latter motivates the high resolution spectroscopic study of $\mathrm{Yb}$ atoms in solid $\mathrm{Ne}(\mathrm{sNe})$ presented here.

Recent work at relatively low resolution $(\sim 0.5 \mathrm{~nm})$ has detailed some of the complexities of $\mathrm{Yb} / \mathrm{RG}$ systems. When solid Xe was used as the matrix host, absorption and emission bands were found to have a two-fold structure due to $\mathrm{Yb}$ occupation in tetravacancies and single substitutional sites [15]. Earlier work found that when solid Ar was the matrix host, the same bands had a threefold structure, due to $\mathrm{Yb}$ occupation in hexavacancies, tetravacancies and single substitutional sites [16]. The symmetry of these trapping sites generally induces crystal field splitting (CFS) of degenerate atomic states upon excitation [17. Low symmetries further multiply the number of bands, while high symmetries create secondary structures due to Jahn-Teller electron-phonon coupling [18. The phonon structure itself is usually not visibleexcept on certain forbidden transitions of the $\mathrm{Mn} / \mathrm{Kr}[19]$ and $\mathrm{Eu} / \mathrm{Ar}[20$ ] systems - and predominantly contributes to the width of strongly non-Frank-Condon bright absorptions.

The above hierarchy of matrix-induced spectral perturbations is typical of the heavy "classical" RG solids made of $\mathrm{Ar}$, $\mathrm{Kr}$ or Xe. In "quantum" matrices, such as those of $\mathrm{He}$ and $\mathrm{H}_{2}$, for which the effects of nuclear motion are expected to be much stronger, the identity of distinct trapping sites is eroded and large-amplitude motions magnify electron-nuclear couplings. Solid Ne is often regarded as a "semi-quantum" crystal in which one may expect incipient nuclear quantum effects 21. At the same time, the Ne crystal is a less perturbing envi- ronment than the heavier RGs. Isolated in it, the $\mathrm{Yb}$ electronic structure is still qualitatively the same as in vacuum, while the lifetimes of excited states are not significantly different 10, 11. Based on these observations, single atom detection of $\mathrm{Yb}$ in sNe would appear feasible, which could then be extended for use in detecting rare nuclear reactions 22. This would be in close analogy with the recent demonstration of single atom imaging of $\mathrm{Ba}$ in solid Xe, performed with a view to searching for neutrinoless double beta decay 23$]$.

In order to investigate all these prospects, we have performed the first high resolution $(0.025 \mathrm{~nm})$ broadband spectroscopy of the low-lying states of the $\mathrm{Yb} / \mathrm{Ne}$ system (Fig. 1). Five absorption bands were identified as due to $\mathrm{Yb}$ atomic transitions from the ${ }^{1} S_{0}$ ground state of the $4 f^{14} 5 d^{0} 6 s^{2}$ configuration to $J=1$ states of the $4 f^{14} 5 d^{0} 6 s 6 p$ or $4 f^{13} 5 d^{1} 6 s^{2}$ configuration. These appear as either broad, largely featureless bands or narrow, highly structured ones depending on their susceptibility to the matrix-induced perturbations described above. To assist in their interpretation, we performed classical simulations that gave the structure and symmetry of the $\mathrm{Yb} / \mathrm{Ne}$ trapping sites and whose corresponding theoretical spectra adequately explained the observed lineshapes. The spectrum resulting from steady state $6 s^{2}{ }^{1} S_{0} \rightarrow 6 s 6 p{ }^{1} P_{1}$ excitation was also recorded at the same high resolution. Knowledge of the decay paths originating from the excited singlet state will be important for single atom spectroscopy.

The remainder of this paper is organized as follows. Section [II details the experimental methods, while Section III presents the results. These results, together with the classical simulations, are discussed in Section IV. A summary and concluding remarks are made in Section V.

\section{EXPERIMENTAL SETUP}

All the experiments were conducted with the liquid helium cryostat illustrated in Fig. 2. Its cold surface has an area of $300 \mathrm{~cm}^{2}$ that is cooled by the liquid helium bath to $4.2 \mathrm{~K}$, at which temperature the pressure of the vacuum falls below $10^{-8}$ Torr. The $\mathrm{Yb} / \mathrm{Ne}$ samples were grown on a $2.54 \mathrm{~cm}$ diameter c-plane sapphire substrate installed vertically on the cold surface using a copper mount. Indium wires and low vapor pressure grease (Apiezon N) were used to achieve good thermal contact between the substrate and the cold surface. The temperature of the substrate was measured by a resistance temperature detector (Lakeshore CX-1050-AA) and was typically at 4.2-5.0 $\mathrm{K}$ depending on the thermal contact.

There were four $2.54 \mathrm{~cm}$ diameter ports on the perimeter of the cryostat. One port was connected to an effusion oven whose crucible was loaded with metallic Yb of natural isotope abundance. The other three ports (A, B and C in Fig. 2 were closed with fused silica windows and used as viewports. Viewports $\mathrm{A}$ and $\mathrm{B}$, which were each at an angle of $22.5^{\circ}$ to the normal direction of the substrate 


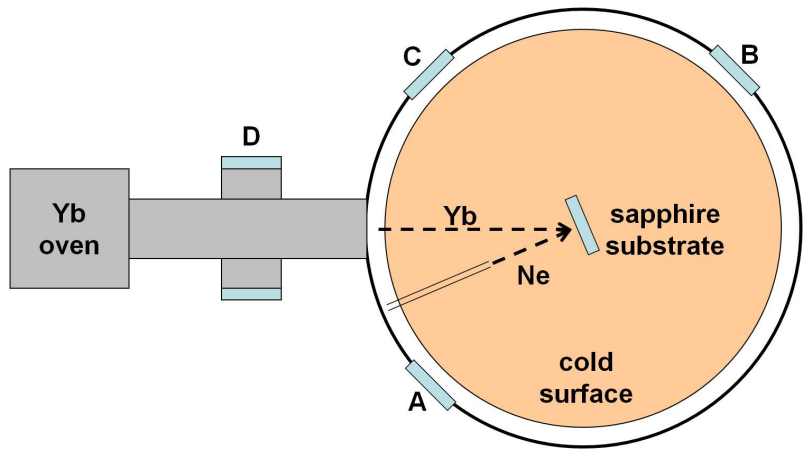

FIG. 2: A sketch of the cold surface inside the liquid helium cryostat together with the effusion oven and $\mathrm{Yb}$ beam line. $\mathrm{A}, \mathrm{B}$ and $\mathrm{C}$ are three viewports covered with fused silica windows. $\mathrm{D}$ is a six-way cross where the $\mathrm{Yb}$ beam intensity is measured.

surface, were used during measurements of the sample thickness and the absorption spectroscopy. Viewport C, which was at an angle of $67.5^{\circ}$ to the normal direction of the substrate surface, was used in the emission study to collect fluorescence from the sample upon excitation.

The sample was prepared as follows: the Ne gas first flowed through a purifier (LDetek LDP1000) and a 77 $\mathrm{K}$ charcoal trap and then leaked into the cryostat via a capillary tube that ended $5 \mathrm{~cm}$ away from the substrate. The implantation of $\mathrm{Yb}$ atoms was achieved by directing the beam from the effision oven at the substrate while the sNe crystal grew. The sample thickness was determined by recording the transmitted light of a He-Ne laser and counting the fringes due to the thin-film interference effect. Meanwhile, the $\mathrm{Yb}$ intensity was measured using the cross-beam-fluorescence method at the six-way cross (viewport D in Fig. 2). A $398.9 \mathrm{~nm}$ laser beam resonant with the $\mathrm{Yb} 6 s^{2}{ }^{1} S_{0} \rightarrow 6 s 6 p{ }^{1} P_{1}$ transition in vacuum was applied perpendicularly to the $\mathrm{Yb}$ beam and the fluorescence measured using a photomultiplier tube along an axis perpendicular to both beams.

We found empirically that individual $\mathrm{Yb}$ atoms (as opposed to higher aggregates) were isolated in sNe only when the $\mathrm{Yb}$ :Ne ratio was kept below $5 \mathrm{ppm}$. In practice, we held that ratio at $1 \mathrm{ppm}$ for which a $\mathrm{Yb}$ beam intensity of $4 \times 10^{14}$ atoms $/ \mathrm{cm}^{2} \cdot \mathrm{hr}$ and an oven temperature of $620 \mathrm{~K}$ were required. The steady state Ne growth rate was typically $50 \mu \mathrm{m} / \mathrm{hr}$ and the $\mathrm{Yb}$ areal and volumetric densities were estimated to be $2 \times 10^{15}$ atoms $/ \mathrm{cm}^{2}$ and $10^{16}$ atoms $/ \mathrm{cm}^{3}$ respectively. Under these conditions, the heat load due to the deposition of $\mathrm{Ne}$ and the blackbody radiation of the oven increased the substrate temperature by only $50 \mathrm{mK}$. A ramp-up time of two hours was required to preserve the transparency of the sample and, once this rate was reached, it could be grown for five hours before it cracked under its own internal strain.

In the absorption study, a weak broadband light source (Ocean Optics DH2000-DUV) illuminated the sample through viewport $\mathrm{A}$, and the transmitted light was collected through viewport $\mathrm{B}$ and fiber-coupled to a spectrometer. In the emission study, a light-emitting diode (LED) centered at $385 \mathrm{~nm}$ illuminated the sample through viewport $\mathrm{A}$, and the induced fluorescence was collected at viewport $\mathrm{C}$ and also fiber-coupled to a spectrometer. Two spectrometers were available to us to analyze the absorption and the emission signals, both of which used line CCD cameras as the detectors. The Ocean Optics USB4000-UV-VIS spectrometer covered a broad range from $200 \mathrm{~nm}$ to $1100 \mathrm{~nm}$ and had a resolution of $1.5 \mathrm{~nm}$. The McPherson 225 spectrometer had a resolution of $0.025 \mathrm{~nm}$ and covered a range from $0 \mathrm{~nm}$ to $600 \mathrm{~nm}$ but with a camera frame that was only $40 \mathrm{~nm}$ wide.

To obtain the highest possible spectral resolution, we also used an optical parametric oscillator (OPO) (Continuum Sunlite EX) as the probe light. The OPO was pumped by a third harmonic pulsed Nd:YAG laser (Continuum Powerlite DLS) before passing through a frequency doubler (Inrad Autotracker II) to provide UV light. Each pulse was about $10 \mathrm{~ns}$ long and contained about $1 \mathrm{~mJ}$ of energy. The light was attenuated by four orders of magnitude to avoid melting the sample and damaging the detector. The output beam was split into a probe beam that went through the sample and a reference beam that traveled outside the cryostat. The linewidth of the OPO was about $1 \mathrm{GHz}$ and the frequency was scanned in $2 \mathrm{GHz}$ steps to map out the lineshape of the whole absorption band.

\section{RESULTS}

\section{A. Probe with Broadband Light}

Figures 3 and 4 show the white-light absorption spectra of a typical sample taken, respectively, with the 1.5 $\mathrm{nm}$ resolution Ocean Optics and $0.025 \mathrm{~nm}$ McPherson spectrometers. Sample deposition leads to a decrease in the transparency of the subtrate which manifests itself as frequency-dependent distortions in the baselines. Nonetheless, five absorption bands, represented in terms of absorbance, can be clearly seen and are identified as due to electronic transitions originating from ground state $\mathrm{Yb}$ atoms. Given that the configuration of the ground state is $4 f^{14} 5 d^{0} 6 s^{2}$, low-lying excitations can either be $6 s \rightarrow 6 p$ outer-shell transitions or $4 f \rightarrow 5 d$ innershell transitions, the latter of which break the full $4 f$ shell and leave the $6 s$ shell intact. In the spectra, bands (a) and (b) correspond to outer-shell transitions whose final levels are $6 s 6 p{ }^{3} P_{1}$ and $6 s 6 p{ }^{1} P_{1}$ respectively. In contrast, bands (c), (d) and (e) correspond to inner-shell transitions and their final states are $\left[4 f^{13}\right]_{j_{1}}\left[5 d^{1}\right]_{j_{2}} 6 s^{2}$ in the $j j$ coupling scheme with $\left(j_{1}, j_{2}\right)_{J}$ equal to $(7 / 2,5 / 2)_{1}$, $(5 / 2,5 / 2)_{1}$ and $(5 / 2,3 / 2)_{1}$, respectively. A comparison between the wavenumbers of transitions in vacuum and those in sNe is summarized in Table \.

Band (a), assigned to the intercombination $6 s^{2}{ }^{1} S_{0} \rightarrow$ $6 s 6 p{ }^{3} P_{1}$ transition, was not observed in our earlier 


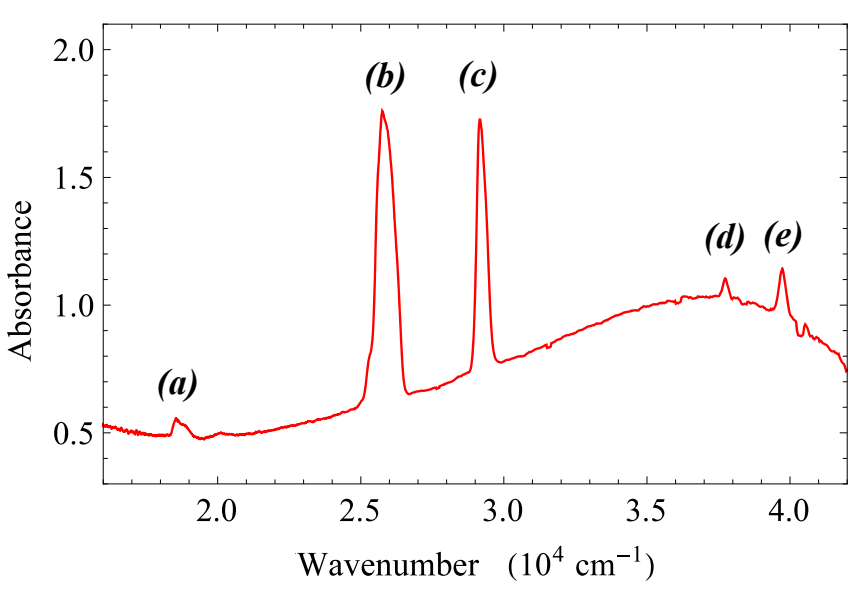

FIG. 3: The absorption spectrum of $\mathrm{Yb}$ atoms in solid $\mathrm{Ne}$ taken by the Ocean Optics spectrometer with $1.5 \mathrm{~nm}$ resolution. All five absorption peaks are identified as $\mathrm{Yb}$ atomic transitions from the ground state. See Table $\mathrm{I}$ for details.

work but becomes visible here due to an increase in the $\mathrm{Yb}$ areal density by an order of magnitude 10 . Another difference is in our reassignment of band (d). Recent spectroscopic data and calculations of free $\mathrm{Yb}$ atoms [24 26] indicate that it should be associated the $4 f^{13} 5 d^{1} 6 s^{2}(5 / 2,5 / 2)_{1}$ state rather than, as previously thought, the $4 f^{13} 5 d^{2} 6 s^{1}$ one. In the case of band (e), there was also some ambiguity in its assignment originally. In earlier work it has been assigned to to the excited $6 s 7 p{ }^{1} P_{1}$ level, the energy of which is $40,564 \mathrm{~cm}^{-1}$ above the ground state in vacuum [27]. This implies a red-shift in its transition frequency in sNe that is peculiar given that the other four transitions are all blue-shifted and makes the narrowness of its peaks inconsistent with the typical effects of the matrix on an $s \rightarrow p$ transition. Reassigning it to the $4 f^{13} 5 d^{1} 6 s^{2}(5 / 2,3 / 2)_{1}$ level removes these inconsistencies, though at the cost of a larger-thanexpected absolute shift.

In matrix isolation, outer-shell and inner-shell transitions have different lineshapes due to the screening of medium effects by the outer-shell electrons 27. For $\mathrm{Yb}$ in sNe, the difference can be readily seen by comparing bands (a) and (b) (6s $\rightarrow 6 p)$ with bands (c), (d) and

TABLE I: The assignment of the absorption bands appearing in Fig. 3 Here, $\sigma_{0}$ represents the wavenumbers of the transitions in vacuum, $\sigma_{\mathrm{abs}}$ the wavenumbers of the transitions in solid Ne, and $\Delta \sigma$ the wavenumber shifts. The wavenumbers are in $\mathrm{cm}^{-1}$ with an uncertainty of $\pm 40 \mathrm{~cm}^{-1}$ in solid Ne.

\begin{tabular}{|c|c|c|c|c|}
\hline$\overline{\text { Band }}$ & Transition & $\sigma_{0}$ & $\sigma_{\mathrm{abs}}$ & $\Delta \sigma$ \\
\hline (a) & $6 s^{2}{ }^{1} S_{0} \rightarrow 6 s 6 p^{3} P_{1}$ & 17,992 & 18,510 & +518 \\
\hline (b) & $6 s^{2}{ }^{1} S_{0} \rightarrow 6 s 6 p{ }^{1} P_{1}$ & 25,068 & 25 & +692 \\
\hline (c) & $6 s^{2}{ }^{1} S_{0} \rightarrow 4 f^{13} 5 d^{1} 6 s^{2}(7 / 2,5 / 2)_{1}$ & 28,857 & 29,140 & +283 \\
\hline (d) & $6 s^{2}{ }^{1} S_{0} \rightarrow 4 f^{13} 5 d^{1} 6 s^{2}(5 / 2,5 / 2)_{1}$ & 37,415 & 37,730 & +315 \\
\hline (e) & $6 s^{2}{ }^{1} S_{0} \rightarrow 4 f^{13} 5 d^{1} 6 s^{2}(5 / 2,3 / 2)_{1}$ & 38,422 & 39,680 & $+1,260$ \\
\hline
\end{tabular}

(e) $(4 f \rightarrow 5 d)$ at the higher spectral resolution of Figure 4. Band (a) has two principal structures with maxima at $18,530 \mathrm{~cm}^{-1}$ and $18,826 \mathrm{~cm}^{-1}$, while band (b) has three principal structures with maxima at $25,525 \mathrm{~cm}^{-1}$, $25,724 \mathrm{~cm}^{-1}$ and $25,774 \mathrm{~cm}^{-1}$. Although both bands (a) and (b) display some splitting, the linewidth of each peak remains a few hundred wavenumbers. By contrast, bands (c), (d) and (e) have more complex structures in which the narrowest linewidth is comparable to the instrument resolution. The three principal structures in band (c) have their peaks at $28,988 \mathrm{~cm}^{-1}, 29,049 \mathrm{~cm}^{-1}$ and $29,073 \mathrm{~cm}^{-1}$. In band (e) there are two clear peaks at $39,631 \mathrm{~cm}^{-1}$ and $39,652 \mathrm{~cm}^{-1}$ and an unresolved shoulder extending from 39,580 to $39,610 \mathrm{~cm}^{-1}$. In band (d) we have a distinct doublet with peaks at $37,741 \mathrm{~cm}^{-1}$ and $37,751 \mathrm{~cm}^{-1}$ and a series of smaller peaks constituting a substructure that extends to lower frequencies.

In the subsequent sections, we propose that the principal peaks of these latter bands are connected to ZPLs on individual crystal field levels. We also propose that substructures on these bands are the manifestations of phonon excitations that produce the phonon sidebands typically associated with ZPLs, of which band (d) offers a prime example.

\section{B. Probe with Laser Light}

In order to investigate further the vibronic progression seen in band (d), the $6 s^{2}{ }^{1} S_{0} \rightarrow 4 f^{13} 5 d^{1} 6 s^{2}(5 / 2,5 / 2)_{1}$ transition was probed at $2 \mathrm{GHz}$ resolution using frequency doubled light from the OPO. Figure 5 shows a plot of the absorbed light intensity (normalized against the incident light intensity) versus wavenumber. As with the original white-light spectra, the two peaks centered at $37,741 \mathrm{~cm}^{-1}$ and $37,751 \mathrm{~cm}^{-1}$ were assigned to the $4 f^{13} 5 d^{1} 6 s^{2}(5 / 2,5 / 2)_{1}$ state. Extending to lower frequencies, between $37,631 \mathrm{~cm}^{-1}$ and $37,733 \mathrm{~cm}^{-1}$, one can see a series of 9 smaller, consecutive peaks. Possible explanations for this series include multiple site occupancy and/or CFS. However, both of these seem unlikely in this case. First, there is no analogous structure in bands (c) and (e), which indicates that this is a phenomenon peculiar to the $4 f^{13} 5 d^{1} 6 s^{2}(5 / 2,5 / 2)_{1}$ transition. Second, attributing this series to multiple site occupancy would require the existence of too many additional trapping sites: at least 3 of axial symmetry, given that CFS gives rise to 3 features for each $J=1$ state; and at most 9 , if we assume a one-to-one correspondence between the peaks and trapping sites. This is unsupported by the theoretical calculations of Section IVA, while the regularity of the peaks - a spacing of $14 \mathrm{~cm}^{-1}$ for the first four - has never been observed in other cases of multiple site occupation.

Instead, we interpret the harmonic series of Fig. 5 as evidence of coupling between the electronic transition and a small number of local phonon modes. This type of structure is usually not visible in RG matrices as 


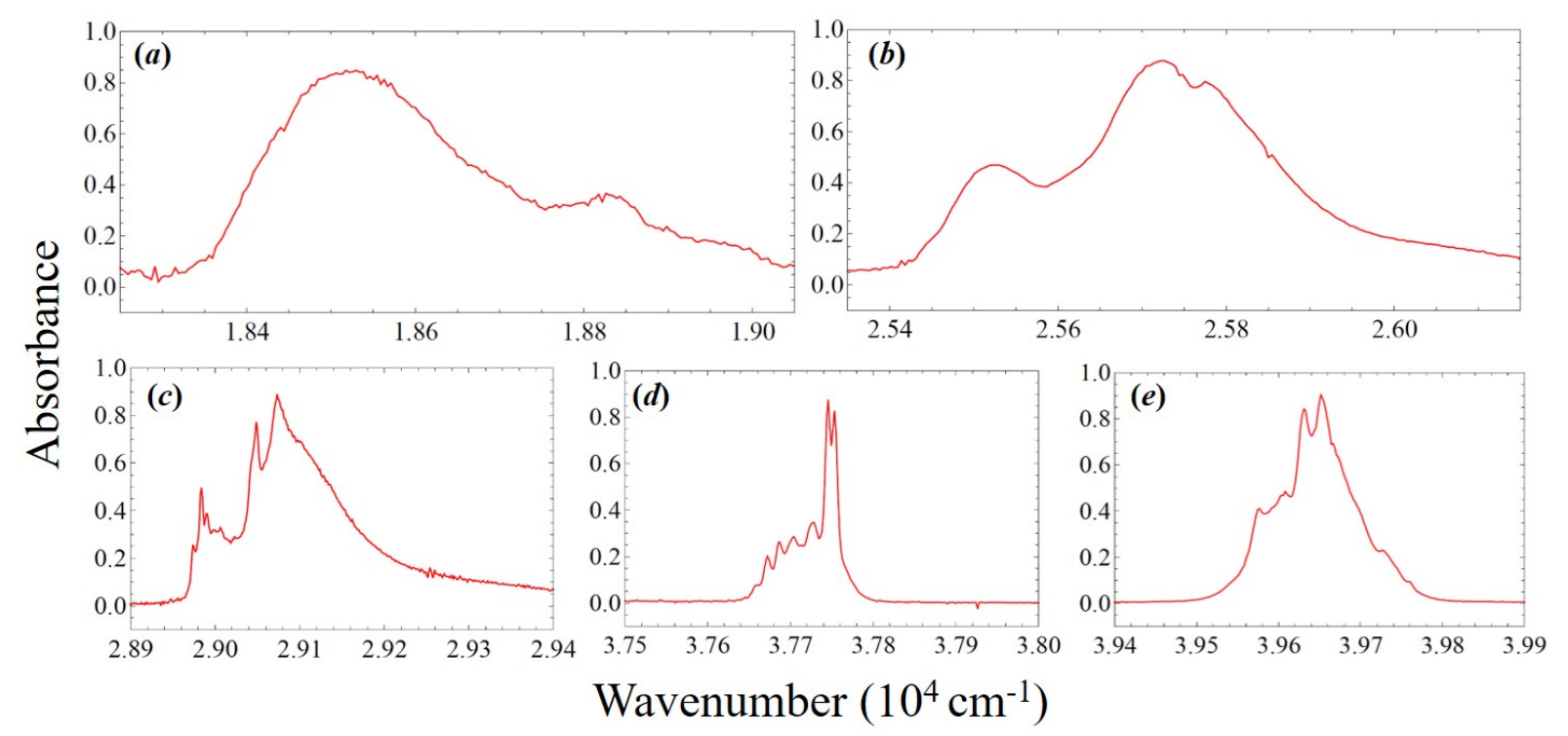

FIG. 4: The detailed lineshapes of the absorption bands shown in Fig. 3 registered using the McPherson spectrometer at 0.025 nm resolution. The labels (a)-(e) are the same as those used in Table I

electronic transitions tend to couple strongly to a large number of lattice modes to produce broad unstructured bands in the spectra. In ionic crystals, the emergence of strong vibronic sidebands in intra $4 \mathrm{f}^{N}$ electronic transitions is associated with strong configuration interaction and coupling to charge transfer states [28. These mechanisms likely also operate here since crystal field splitting induced by van der Waals forces in the neutral $\mathrm{Mn} / \mathrm{Kr}$ system is attributed to the dominance of: (i) inter-electronic repulsion in the metal atom in the case of weak splitting; or (ii) ligand-metal electron repulsion in the case of strong splitting [19].

In the most common treatment, the strength of the electron-phonon coupling is measured by the HuangRhys parameter, $S$, which is related to the displacement in the lattice equilibrium position near a defect site, $\Delta$, accompanying an electron transition according to:

$$
S=\frac{4 \beta^{2}}{\left(1+\beta^{2}\right)^{2}} \frac{\omega M \Delta^{2}}{2 \hbar}, \beta^{2}=\frac{\omega^{\prime}}{\omega}
$$

for which $\omega$ and $\omega^{\prime}$ are the frequencies of the vibrational mode in the initial and final electronic states, respectively, and $M$ is the effective mass of the defect site. At very low temperature (i.e. $\hbar \omega>k T \sim 2.8-3.5 \mathrm{~cm}^{-1}$ ) vibronic transitions can be approximated as arising from the zero-phonon $(n=0)$ state and $p_{n}$, the probability that the $n$th vibrational state is populated, can be straightforwardly shown under the Franck-Condon approximation to obey $p_{n}(S) \propto \exp (-S) S^{n} / n$ ! which is also the form of the overall envelope of the vibronic band [29. Within that band, on the assumption that coupling involves a single vibrational mode and that the frequencies of the oscillators follow a Gaussian distribution around the mode's average frequency, $\omega$, the intensity function of the phonon line $n$ is [30]:

$$
\begin{aligned}
f_{n}(E) & =\exp (-S) \frac{S^{n}}{n !} \\
& \times \frac{1}{\sqrt{2 \pi n \sigma^{2}}} \exp \left(\frac{-\left[E-\left(E_{0}+n \hbar \omega\right)\right]^{2}}{2 n \sigma^{2}}\right),
\end{aligned}
$$

for which $\sigma \sqrt{n}$ is its linewidth and $E_{0}$ is the energy difference between the initial and final electronic states.

We therefore expect to see a harmonic progression in the absorption spectrum in intervals of $\hbar \omega$ and, in Fig. 5. the three phonon lines corresponding to $n=1,2,3$ can be clearly distinguished, centered at $37,655 \mathrm{~cm}^{-1}$, $37,669 \mathrm{~cm}^{-1}$ and $37,683 \mathrm{~cm}^{-1}$, respectively. Per Equation (2), the $n=0$ order corresponding to the ZPL should be a $\delta$-function. In reality, because of electronic dispersion and inhomogeneous broadening due to trapping site inhomogeneity it has a finite width, $\sigma_{0}$. Higher order $(n>3)$ phonon lines are also visible in the range of $37,690 \mathrm{~cm}^{-1}$ to $37,733 \mathrm{~cm}^{-1}$, though they are not so easily resolved, possibly because the single phonon approximation no longer holds and interference between multiple modes should be considered. For the sake of simplicity, we only fit the $n=0-3$ orders using a function for the absorption intensity, $A(E)$, that superimposes the ZPL on the single frequency mode harmonic progressing band 31]:

$$
\begin{aligned}
A(E) & =A_{0} \sum_{n=0}^{n=3} \exp (-S) \frac{S^{n}}{n !}\left(\frac{E_{0}+n \hbar \omega}{E_{0}}\right) \\
& \times \frac{1}{\sqrt{2 \pi\left(\sigma_{0}^{2}+n \sigma^{2}\right)}} \exp \left(-\frac{\left[E-\left(E_{0}+n \hbar \omega\right)\right]^{2}}{2\left(\sigma_{0}^{2}+n \sigma^{2}\right)}\right)+O .
\end{aligned}
$$




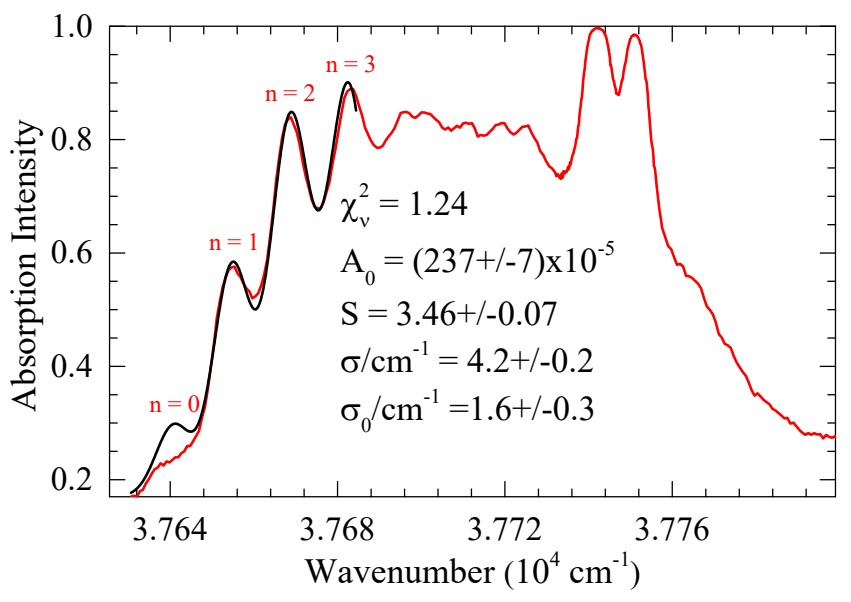

FIG. 5: Absorption intensity of the $6 s^{2}{ }^{1} S_{0} \rightarrow$ $4 f^{13} 5 d^{1} 6 s^{2}(5 / 2,5 / 2)_{1}$ transition using the frequency doubled $\mathrm{OPO}$ as the probe light. The spectrum has a resolution of 2 $\mathrm{GHz}$. The parameters obtained by using Equation (3) to fit the first four features of the vibronic band - indicated by the black line - are given inset.

The small fixed offset $O=A\left(E=37,630 \mathrm{~cm}^{-1}\right)=$ 0.1703 accounts for the non-zero background in Fig. 5 produced by light scattered from the matrix. The same figure gives the phonon and ZPL frequencies as $\omega=14.0$ $\mathrm{cm}^{-1}$ and $E_{0}=37,641 \mathrm{~cm}^{-1}$, respectively. The black trace shows that the fit was performed in the range of 37,630 to $37,685 \mathrm{~cm}^{-1}$ and gives the following values for the parameters: $A_{0}=(237 \pm 7) \times 10^{-5}, S=3.46 \pm 0.07$, $\sigma_{0}=1.6 \pm 0.3 \mathrm{~cm}^{-1}$ and $\sigma=4.2 \pm 0.2 \mathrm{~cm}^{-1}$. The lattice displacement, $\Delta$, can be calculated using Equation (1) under the approximations that $\beta \simeq 1$ (i.e. $\omega \simeq \omega^{\prime}$ ) and $\bar{M}$ is equal to the sum of the masses of the $\mathrm{Yb}$ atom and its nearest neighbor Ne atoms. We thus obtain $\Delta=1.29 \pm$ $0.01 \AA$, which is approximately $25 \%$ of the ground-state $\mathrm{Yb}-\mathrm{Ne}$ bond length. This displacement is quite realistic and supports our value for $S$. It can be easily seen that the fit is least accurate for the $n=0$ feature. The ZPL at $E_{0}=37,641 \mathrm{~cm}^{-1}$ is only minimally visible because its intensity is reduced in favor of the phonon sideband. It should be more prominent at lower temperatures. The maxima of the peaks at $37,741 \mathrm{~cm}^{-1}$ and $37,751 \mathrm{~cm}^{-1}$ cannot be completely discerned due to saturation of the detector. However, their sharpness in Fig. 3 (d) strongly suggests that they are also ZPLs.

\section{Emission Spectroscopy}

In earlier work, we showed that strong fluorescence of $\mathrm{Yb}$ atoms trapped in sNe can be induced by driving the $6 s^{2}{ }^{1} S_{0} \rightarrow 6 s 6 p{ }^{1} P_{1}$ transition [11, 32]. Both a $1 \mathrm{MHz}$ linewidth laser centered at $388 \mathrm{~nm}\left(25,770 \mathrm{~cm}^{-1}\right)$ and a $10 \mathrm{~nm}$ linewidth LED centered at $385 \mathrm{~nm}\left(25,970 \mathrm{~cm}^{-1}\right)$ were able to resonantly excite this transition. This results in a significant population transfer to the metastable
$6 s 6 p{ }^{3} P_{0}$ level via $6 s 6 p{ }^{1} P_{1} \rightarrow 6 s 5 d^{3} D_{J}$ intersystem crossing, the branching ratio of which is enhanced by level mixing induced by the crystal field of the matrix. Due to the long lifetime of the $6 s 6 p{ }^{3} P_{0}$ state in $\mathrm{sNe}$, excited $\mathrm{Yb}$ atoms continue absorbing photons to make transitions from this state to even higher excited ones. A strong absorption peak centered at $26,740 \mathrm{~cm}^{-1}$, corresponding to the $6 s 6 p{ }^{3} P_{0} \rightarrow 6 p^{2}{ }^{3} P_{1}$ excitation, has been previously reported [10. Given the broad linewidths of absorption peaks in the solid state, the $385 \mathrm{~nm}$ LED can also excite this transition and emission from states at higher energies than the exciting photons are thus observed.

Figure 6 shows the full steady state emission spectrum of $\mathrm{Yb}$ atoms in sNe excited by the $385 \mathrm{~nm} \mathrm{LED} \mathrm{and}$ recorded at a resolution of $0.025 \mathrm{~nm}$. We divide the emission lines into the three wavenumber regions given below and summarize those we were able to identify in Table II.

Region $1\left(1.7 \sim 2.1 \times 10^{4} \mathrm{~cm}^{-1}\right)$ : The prominent emission lines in this region are responsible for a green glow from the sample when its is pumped by the LED. They include the decays of the fine-structure triplet group $6 s 6 p{ }^{3} P_{0,1,2} \rightarrow 6 s^{2}{ }^{1} S_{0}$ whose spacing in sNe is approximately the same as in the free atom. Although metastable in vacuum, the $6 s 6 p{ }^{3} P_{0,2}$ levels decay radiatively in matrix isolation due to a Stark-like mixing induced by the crystal field. The assignments of the $6 s 6 p{ }^{3} P_{0,1}$ states have been validated by lifetime measurements in earlier work [11. It should be noted that the strong emission line at $18,900 \mathrm{~cm}^{-1}$ does not belong to this triplet group but corresponds to the $6 p^{2}{ }^{3} P_{1} \rightarrow$ $6 s 6 p{ }^{1} P_{1}$ decay following the $6 s 6 p{ }^{3} P_{0} \rightarrow 6 p^{2}{ }^{3} P_{1}$ excitation mentioned above [10]. The emission line at 20,710 $\mathrm{cm}^{-1}$ is the most prominent one that remains unidenti-

TABLE II: The assignment of identifiable emission lines appearing in Fig. 6. Transitions forbidden in vacuum are indicated by the symbol $\uparrow$. Five of the emissions lines are correlated to absorption lines listed in Table $\mathrm{I}$ and are labeled with the same letters. Here, $\sigma_{0}$ represents the wavenumbers of transitions in vacuum, $\sigma_{\text {emis }}$ the wavenumbers of the transitions in solid Ne, and $\Delta \sigma$ the wavenumber shifts. The wavenumbers are in $\mathrm{cm}^{-1}$ with an uncertainty of $\pm 40 \mathrm{~cm}^{-1}$ in solid Ne.

\begin{tabular}{|c|c|c|c|c|}
\hline Transition & & $\sigma_{0}$ & $\overline{\sigma_{\text {emis }}}$ & $\Delta \sigma$ \\
\hline $6 s 6 p{ }^{3} P_{0} \rightarrow 6 s^{2}{ }^{1} S_{0}$ & $(i)^{\dagger}$ & 17,288 & 17,730 & +440 \\
\hline $6 s 6 p{ }^{3} P_{1} \rightarrow 6 s^{2}{ }^{1} S_{0}$ & $(i i) /(a)$ & 17,992 & 18,320 & +320 \\
\hline $6 p^{2}{ }^{3} P_{1} \rightarrow 6 s 6 p{ }^{1} P_{1}$ & (iii) & 18,737 & 18,900 & +160 \\
\hline $6 s 6 p{ }^{3} P_{2} \rightarrow 6 s^{2}{ }^{1} S_{0}$ & $(i v)^{\dagger}$ & 19,710 & 20, & +370 \\
\hline $4 f^{13} 5 d^{1} 6 s^{2}(7 / 2,3 / 2)_{2} \rightarrow 6 s^{2}{ }^{1} S_{0}$ & $(v)^{\dagger}$ & 23,189 & 23,070 & -120 \\
\hline $6 s 5 d{ }^{3} D_{1} \rightarrow 6 s^{2}{ }^{1} S_{0}$ & $(v i)^{\dagger}$ & 24,489 & 24 & $\sim 0$ \\
\hline $6 s 6 p{ }^{1} P_{1} \rightarrow 6 s^{2}{ }^{1} S_{0}$ & $(v i i) /(b)$ & 25,068 & 25,320 & +250 \\
\hline $6 s 5 d{ }^{1} D_{2} \rightarrow 6 s^{2}{ }^{1} S_{0}$ & $(\text { viii })^{\dagger}$ & 27,678 & 27,860 & +180 \\
\hline $4 f^{13} 5 d^{1} 6 s^{2}(7 / 2,5 / 2)_{1} \rightarrow 6 s^{2}{ }^{1} S_{0}$ & $(i x) /(c)$ & 28,857 & 29,030 & +170 \\
\hline $4 f^{13} 6 s^{2} 6 p(7 / 2,3 / 2)_{2} \rightarrow 6 s^{2}{ }^{1} S_{0}$ & $(x)^{\dagger}$ & 35,197 & 35,590 & +390 \\
\hline $4 f^{13} 5 d^{1} 6 s^{2}(5 / 2,5 / 2)_{1} \rightarrow 6 s^{2}{ }^{1} S_{0}$ & $(x i) /(d)$ & 37,415 & 37,730 & +320 \\
\hline $4 f^{13} 5 d^{1} 6 s^{2}(5 / 2,3 / 2)_{1} \rightarrow 6 s^{2}{ }^{1} S_{0}$ & $(x i i) /(e)$ & 38,422 & 39,610 & $+1,190$ \\
\hline
\end{tabular}




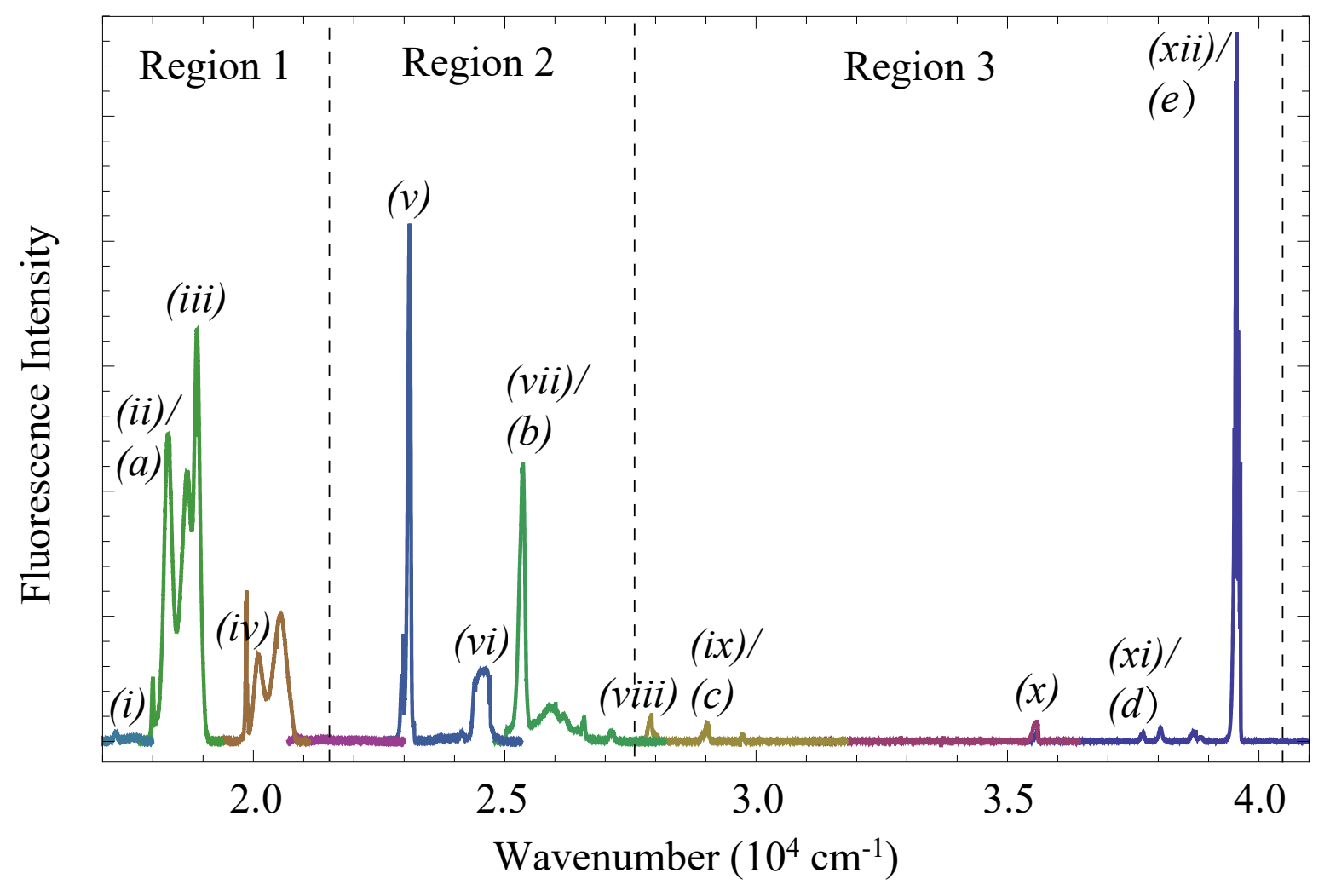

FIG. 6: The steady-state emission spectrum of $\mathrm{Yb}$ atoms in solid Ne induced by the $385 \mathrm{~nm}$ LED and recorded using the McPherson spectrometer at $0.025 \mathrm{~nm}$ resolution. The extent of each color represents the extent of the camera frame. The labels on the identified emission lines are the same as those used in Table II.

fied in this region.

Region $2\left(2.2 \sim 2.6 \times 10^{4} \mathrm{~cm}^{-1}\right)$ : Aside from the $6 s 6 p{ }^{1} P_{1}$ decay at $25,320 \mathrm{~cm}^{-1}$, two strong emission lines are seen in this region. We tentatively propose that the line at $23,070 \mathrm{~cm}^{-1}$ corresponds to the decay of the $4 f^{13} 5 d^{1} 6 s^{2}(7 / 2,3 / 2)_{2}$ state. This excited level has the same electronic configuration as those of absorption peaks (c), (d) and (e), but the $j j$ coupling of $j_{1}=7 / 2$ and $j_{2}=3 / 2$ does not give $J=1$ and its decay is thus forbidden in vacuum. This assignment results in the only negative matrix shift in Table II. However, attempts to assign it to other nearby states, such as $6 s 5 d^{3} D_{1,2}$, result in unnaturally large matrix shifts. On similar grounds, the parity forbidden $6 s 5 d{ }^{3} D_{1} \rightarrow 6 s^{2}{ }^{1} S_{0}$ transition is proposed to be responsible for the $24,490 \mathrm{~cm}^{-1}$ line.

Region $3\left(2.7 \sim 4.0 \times 10^{4} \mathrm{~cm}^{-1}\right)$ : Emission lines in this region have photon energies higher than the exciting photon. We again assign the decay of various excited states according to the proximity of their wavenumbers to those of excited states obtained in vacuum. Thus, the $28,857 \mathrm{~cm}^{-1}$ emission corresponds to $4 f^{13} 5 d^{1} 6 s^{2}(7 / 2,5 / 2)_{1}$ decay; the 37,730 $\mathrm{cm}^{-1}$ emission corresponds to $4 f^{13} 5 d^{1} 6 s^{2} \quad(7 / 2,5 / 2)_{1}$ decay; and the $39,610 \mathrm{~cm}^{-1}$ emission corresponds to
$4 f^{13} 5 d^{1} 6 s^{2}(7 / 2,5 / 2)_{1}$ decay. The profile of the absorption energies that populate these excited states are, respectively, peaks (c), (d) and (e) of Fig. 4. Although the matrix shift of the $6 s^{2}{ }^{1} S_{0} \rightarrow 4 f^{13} 5 d^{1} 6 s^{2}(5 / 2,3 / 2)_{1}$ transition appears rather large, it is comparable to the size of the matrix shift that occurs in the absorption spectrum.

\section{DISCUSSION}

In order to interpret the structure of the absorption bands, we discuss here the consistency of all experimental data invoking also the results of limited theoretical simulations possible for the $\mathrm{Yb} / \mathrm{Ne}$ system.

\section{A. Stable trapping sites}

The thermodynamically stable trapping sites of ground-state atomic $\mathrm{Yb}$ in a perfect face-centered cubic (fcc) Ne crystals were modeled following the approach suggested in our earlier works [15, 16, 33]. The full details of the simulations are summarized in the Supplemental Materials to this paper. In brief, we used $a b$ initio 
$\mathrm{Yb}\left({ }^{1} S_{0}\right)-\mathrm{Ne}\left[32\right.$ and slightly modified Aziz-Slaman $\mathrm{Ne}^{-}$ Ne potentials 34 to represent the pairwise force field. For a large fragment of the $\mathrm{Ne}$ crystal, composed of more than 3,000 atoms, the $\mathrm{Yb}$ accommodation energy, $\Delta E(N)$, was obtained as a function of $N=0,1, \ldots 20$, the number of Ne atoms removed from the crystal. For each $N$, the lowest energy was found by minimization of the trapping site structure in configuration space and corrected by the energy required to remove $N$ crystal atoms.

The meaningful part of the resulting $E(N)$ diagram is shown in Fig. 7. It identifies thermodynamically stable sites as those whose energies lie on its convex hull, in close analogy to the analysis of the discrete variable composition phase diagrams [35. Four such sites are evident: the ground one, having the lowest accommodation energy, with $\mathrm{Yb}$ in an $N=10$ vacancy and three others lying higher in energy with $\mathrm{Yb}$ in $N=8,6$ and 13 vacancies (referred to in what follows as $N \mathrm{~V}$ for " $N$-atom vacancy"). Thus, the Ne crystal tends to accommodate the $\mathrm{Yb}$ atom in quite spacious multiple-atom vacancies. By contrast, $N=4$ and $N=6$ sites compete with each other in solid Ar, an $N=4$ site dominates in solid $\mathrm{Kr}$, and a single substitutional $N=1$ site is the most stable one in solid Xe [15]. Moreover, the energies of the perfect octahedral site, $6 \mathrm{~V}$, and the cuboctahedral site, $13 \mathrm{~V}$, are quite large, while the more energetically stable $10 \mathrm{~V}$ and $8 \mathrm{~V}$ sites have only axial coordination symmetries, $C_{4 v}$ and $C_{2 v}$, respectively (see Supplemental Materials).

These findings have important implications for the CFS of the absorption bands related to $J=1$ level excitations. In the rigid crystal environment of $C_{4 v}$ symmetry, they should appear as two degenerate ZPLs and a single ZPL (with associated substructures if multiple phonons are excited) according to the projection of $J$ onto the site axis $\Omega= \pm 1$ and 0 , falling into $E$ and $A_{1}$ irreducible representations, respectively. The electron-phonon JahnTeller interaction should lift the remaining degeneracy, producing a $2+1$ or $1+2$ generic band structure, as was observed for the axial trapping site of the $\mathrm{Ba}$ atom 36 38. For the site of $C_{2 v}$ symmetry, CFS produces three individual ZPLs.

\section{B. $6 s \rightarrow 6 p$ Absorption Spectra}

The absorption bands due to $6 s \rightarrow 6 p$ transitions in Figs. 4 (a) and (b), appear as broad, weakly structured features, typical of allowed or weakly forbidden outerelectron transitions [19, 36, 39. Upon $s \rightarrow p$ electron promotion, the atom-matrix interaction changes dramatically and becomes strongly anisotropic, so that multiple phonon excitations superimpose relatively large crystal field splittings. At a glance, the $6 s^{2}{ }^{1} S_{0} \rightarrow 6 s 6 p{ }^{3} P_{1}$ and $6 s^{2}{ }^{1} S_{0} \rightarrow 6 s 6 p{ }^{1} P_{1}$ transition profiles of Figs. 4 (a) and 4 (b), respectively, can be interpreted as $2+1$ and $1+2$ split bands originating from the ground $10 \mathrm{~V}$ site.

To verify this interpretation, we simulated the $6 s \rightarrow 6 p$
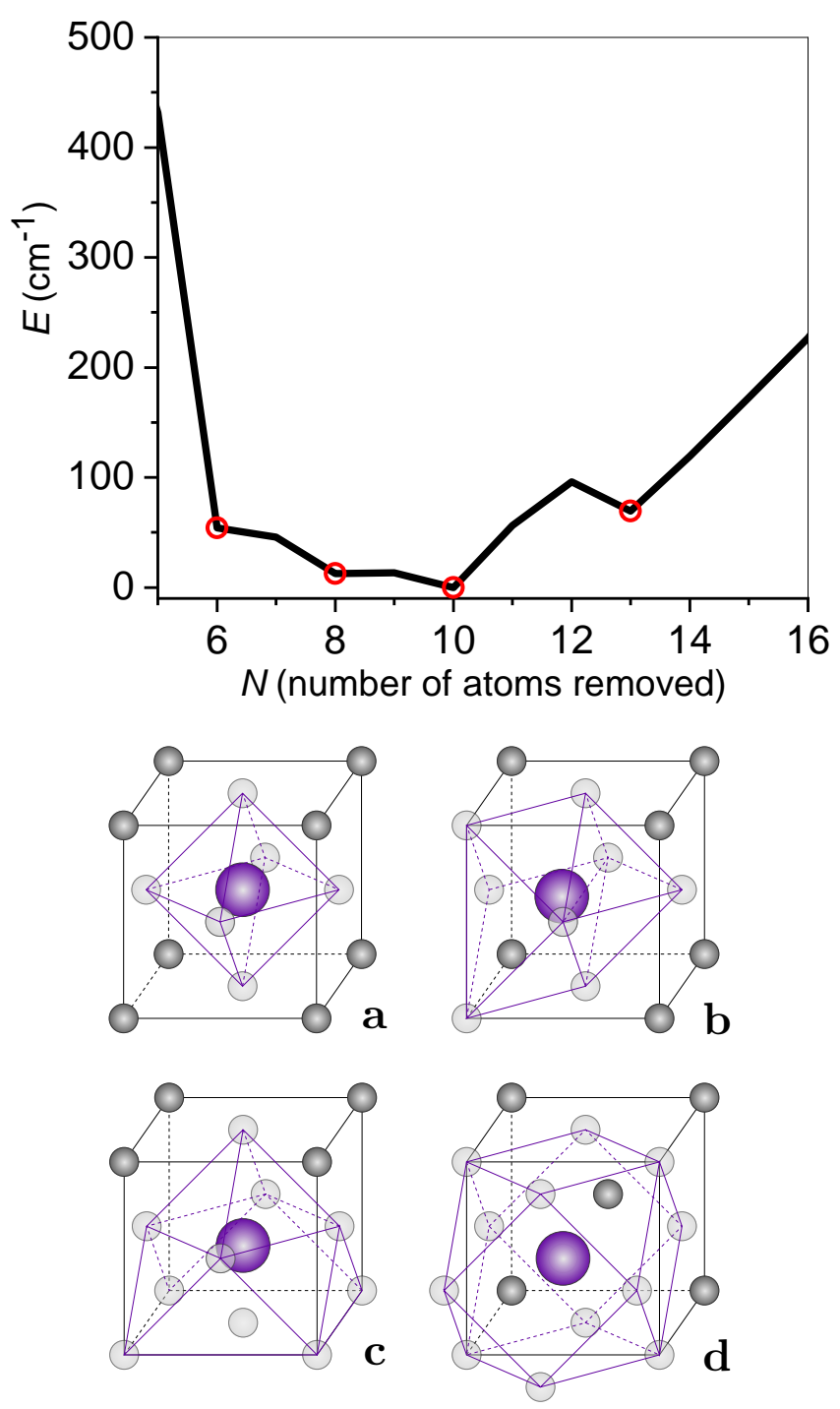

FIG. 7: (color online) Above, the accommodation energy of the $\mathrm{Yb}$ atom in the $\mathrm{Ne}$ fcc crystal as a function of $N$, the number of Ne atoms removed from the lattice (solid line). Red circles indicate the points lying on the convex hull of the $E(N)$ diagram. Below, the schematic structures of the $\mathrm{Yb} / \mathrm{Ne}$ stable trapping sites, 6V (a), 8V (b), 10V (c) and $13 \mathrm{~V}(\mathrm{~d})$. $\mathrm{Yb}$ and $\mathrm{Ne}$ atoms are represented by large and small spheres, respectively; light grey spheres indicate the lattice positions of $\mathrm{Ne}$ atoms removed from the system; dark grey spheres indicate the lattice positions of the remaining $\mathrm{Ne}$ atoms.

absorption spectra using the site geometries found for the ground state and the diatomics-in-molecule model 40 43. parametrized by the $\mathrm{Yb}\left({ }^{3} P_{1},{ }^{1} P_{1}\right)-\mathrm{Ne} a b$ initio potentials available in Ref. 32, For the ${ }^{3} P_{J}$ multiplet, we assumed a weak crystal field limit noting that its spin-orbit coupling constant is on the order of $800 \mathrm{~cm}^{-1}$, while the relative matrix-induced shifts in different types of sites do not exceed $100 \mathrm{~cm}^{-1}$ (Fig. 8). Further details are 


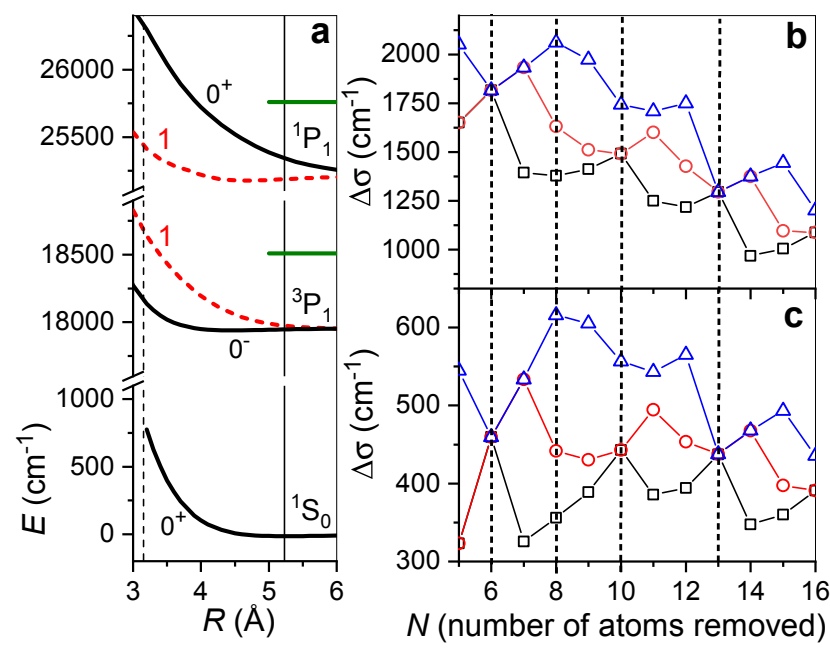

FIG. 8: (color online) (a) Yb-Ne interaction potentials for the ${ }^{3} P_{1}$ and ${ }^{1} P_{1}$ atomic states as functions of the internuclear distance $R$ with $\Omega$ values indicated. The solid vertical line corresponds to the ground-state equilibrium distance, and the dashed vertical line to the Ne-Ne distance in the fcc crystal. The green horizontal bars on the right indicate the maxima of the corresponding absorption bands in Figs. 4. (b) Vertical $6 s^{2}{ }^{1} S_{0} \rightarrow 6 s 6 p^{1} P_{1}$ transition frequency shifts for the lowest energy structures with different $N$. The shifts for each of the excited-state adiabatic PESs relative to the transition frequency in vacuum are presented in black, red and blue colors in ascending order. The vertical dashed lines indicate thermodynamically stable sites. (c) For the $6 s^{2}{ }^{1} S_{0} \rightarrow 6 s 6 p^{3} P_{1}$ transition the same is shown as for the $6 s^{2}{ }^{1} S_{0} \rightarrow 6 s 6 p^{1} P_{1}$ transition in (b).

presented in the Supplemental Materials to this paper. Figure 8 (a) shows the spin-orbit-coupled interaction potentials for the ${ }^{3} P_{1}$ and ${ }^{1} P_{1}$ atomic states. The latter state exhibits much stronger interaction anisotropy-i.e. the difference between the potentials for $\Omega=0$ and \pm 1 components - than the former state. Moreover, the sign of the anisotropy for each state is the opposite of the other state's. Nevertheless, for both states, strong repulsion of one $\Omega$ component overpowers weak attraction of the other $\Omega$ component and leads to an overall positive matrix shift in their absorption frequencies.

Panels (b) and (c) of Fig. 8 present the vertical transition frequency shifts relative to the corresponding atomic transition in vacuum for the lowest energy sites at each $N$. Those corresponding to the stable $6 \mathrm{~V}, 8 \mathrm{~V}, 10 \mathrm{~V}$ and $13 \mathrm{~V}$ sites are marked by vertical lines. Three adiabatic potentials, which correlate to atomic and diatomic terms $(J=1, \Omega)$, split out differently depending on the crystal field symmetry of the site: the polyhedral $6 \mathrm{~V}$ and $13 \mathrm{~V}$ sites maintain threefold degeneracy; the $10 \mathrm{~V}$ site of $C_{4 v}$ symmetry provides a $2+1$ degeneracy pattern; and the $7 \mathrm{~V}$ site of $C_{3 v}$ symmetry, which is unstable for $\mathrm{Yb}$ in solid $\mathrm{Ne}$ but stable for Ba in solid Ar, Kr and Xe 38, produces $1+2$ splitting. In the sites of lower symmetries, degener- acy is completely lifted. Despite anisotropies of opposite signs, the CFS patterns of the singlet and triplet states are qualitatively similar, though the larger splittings in the case of the singlet state reflect its larger interaction anisotropy.

Molecular dynamics simulations were performed for the $6 s \rightarrow 6 p$ absorption bandshapes, as described in the Supplemental Materials. Figure 9 compares the bands simulated for each stable trapping site with bands (a) and (b) of Fig. 4. The simulations systematically overestimate the matrix shift of the $6 s^{2}{ }^{1} S_{0} \rightarrow 6 s 6 p{ }^{1} P_{1}$ absorption and, to a lesser extent, underestimate the shift of the $6 s^{2}{ }^{1} S_{0} \rightarrow 6 s 6 p{ }^{3} P_{1}$ absorption. These errors are attributed to uncertainties in the short-range repulsive branches of the diatomic potentials, which strongly affect the transition frequencies. In agreement with the above analysis of the vertical transition frequencies, the simulated $6 s^{2}{ }^{1} S_{0} \rightarrow 6 s 6 p{ }^{1} P_{1}$ bands are very broad and exhibit a remarkable dependence on the symmetry of the trapping site. The structures seen in their shapes fully agree with the CFS of the vertical transitions: $2+1$ for the $10 \mathrm{~V}$ site with the two-fold degeneracy broken by the Jahn-Teller effect; a symmetric Jahn-Teller triplet for the triply degenerate $6 \mathrm{~V}$ site; and an asymmetric triplet for the $8 \mathrm{~V}$ site. An unstructured asymmetric lineshape is obtained for the polyhedral $13 \mathrm{~V}$ site due to the large dynamical distortion of this spacious and labile structure. By contrast, the simulations of the $6 s^{2}{ }^{1} S_{0} \rightarrow 6 s 6 p{ }^{3} P_{1}$ absorption predict much narrower and strongly overlapping bands. The smaller anisotropy of this state leads to smaller CFS, which is partially washed out for the $8 \mathrm{~V}$ site. The Jahn-Teller structure is not resolved at all and only contributes to the broadening of the bands.

The simulations confirm that the observed $6 s^{2}{ }^{1} S_{0} \rightarrow$ $6 s 6 p{ }^{3} P_{1}$ absorption band bears a $2+1$ CFS structure typical of axially-symmetric sites. It may be due to occupation in a single site, most likely the $10 \mathrm{~V}$ one, or involve contributions from the other trapping sites, which all produce bands of very similar shape, as can be seen in Fig. 9 (a). Interpretation of the ${ }^{1} P_{1}$ absorption is less obvious. Uncertainty in the excited-state $\mathrm{Yb}\left({ }^{1} P_{1}\right)$-Ne potentials is unlikely to be so large as to cause an inverted CFS structure for the 10V site, and we therefore explain the observed $1+2$ structure as a result of multiple site absorptions, which, for this particular transition, produce the distinct band shapes seen in Fig. 9(b). Attempts to fit the observed envelopes of the absorption spectrum to the weighted sum of the simulated ones gave ambiguous results. Excitation spectroscopy, which we reserve for future work, is expected be more informative for the discrimination of the distinct trapping sites.

\section{C. $\quad 4 f \rightarrow 5 d$ Absorption Spectra}

The high-resolution absorption spectra of the $\mathrm{Yb} / \mathrm{Ne}$ system shown in Fig. 4 exhibit a number of features that have not been previously detected in heavier RG 


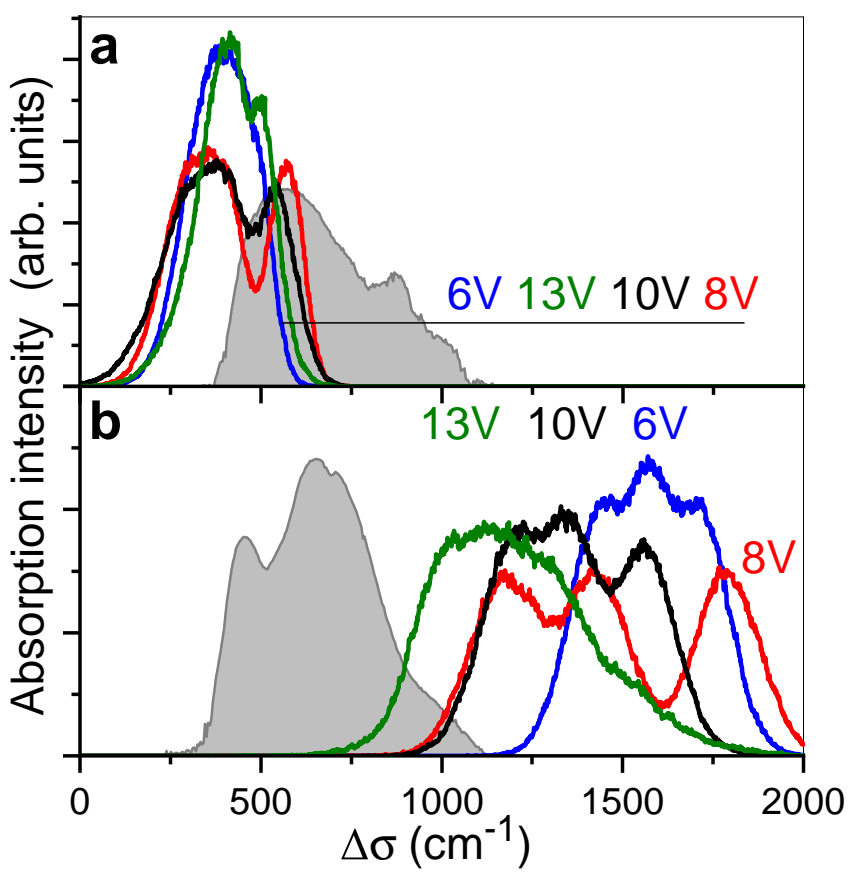

FIG. 9: (color online) Simulated and observed absorption intensities of the ${ }^{3} P_{1}$ (a) and ${ }^{1} P_{1}$ (b) transitions. Frequency is presented in terms of the shift relative to the atomic transition in vacuum. Each band is normalized to unit area.

matrices. In the latter, at low resolution, the spectra are structured in a way that reflects occupation in multiple trapping sites. Here, however, we observe much sharper structures that have qualitative similarities across all three $4 f \rightarrow 5 d$ absorption bands of Figs. 4 (c)-(e). As described in Sec. III A. band (c) has a low frequency peak at $28,988 \mathrm{~cm}^{-1}$ and a high-frequency doublet with peaks at $29,049 \mathrm{~cm}^{-1}$ and $29,073 \mathrm{~cm}^{-1}$; band (e) also has a doublet, with peaks at $39,631 \mathrm{~cm}^{-1}$ and $39,652 \mathrm{~cm}^{-1}$, and a less resolved shoulder to its red from 39,580 to 39,610 $\mathrm{cm}^{-1}$; and in band $(\mathrm{d})$ the doublet has its peaks at 37,741 $\mathrm{cm}^{-1}$ and $37,751 \mathrm{~cm}^{-1}$. The latter band has a long harmonic progression in its red wing, which Fig. 5 shows to be especially well-resolved under laser excitation. We attributed this progression to phonon sidebands and the fit presented in Sec. IIIB fully confirms this assignment. A more erratic secondary structure seen in band (c) may have the same origin, though the spacings between the first four peaks vary from 6 to $10 \mathrm{~cm}^{-1}$.

It is extremely difficult to address the $4 f^{13} 5 d^{1} 6 s^{2}$ states of $\mathrm{Yb}$ by means of $a b$ initio methods and thus perform a theoretical analysis similar to that presented above for the $6 s \rightarrow 6 p$ absorption spectra. Nonetheless, it is possible to make plausible inferences. First, the structure of the atomic states originating from the $4 f^{13} 5 d^{1} 6 s^{2}$ configuration is dominated by $j j$-coupling and shows large spin-orbit splitting 24-26]. The weakfield approximation used for the ${ }^{3} P_{J}$ multiplet should be equally valid. It is thus reasonable to attribute the over- arching band structure to the $1+2$ CFS of the $J=1$ level in the axial crystal environment. As discussed above, simulations indicate that the $10 \mathrm{~V}$ and $8 \mathrm{~V}$ the trapping sites predominate.

Second, it is well-known from experiments in atomic isolation and cooling in magnetic traps and their theoretical interpretation [44, 45] that interaction anisotropy of the open-shell $4 f^{n} 6 s^{2}$ lanthanide atoms with $\mathrm{He}$ is strongly suppressed. As both $4 f$ and $5 d$ shells of the $\mathrm{Yb}$ atom are beneath the outer spherical $6 s^{2}$ shell, the same suppression should be expected for the states arising from the $4 f^{13} 5 d^{1} 6 s^{2}$ configuration. For the same reason, the interaction potentials of these states with RG atoms should be similar to those for the $4 f^{14} 6 s^{2}$ ground state. This accounts for why the peaks in Figs. 4 (c) to (e) are essentially diagonal Frank-Condon envelopes accompanied by phonon overtones. This also explains why the $1+2$ crystal field splittings of the $4 f \rightarrow 5 d$ bands do not exceed $100 \mathrm{~cm}^{-1}$, whereas they are $\sim 200$ and $\sim 300 \mathrm{~cm}^{-1}$ for the outer-shell $6 s^{2}{ }^{1} S_{0} \rightarrow 6 s 6 p{ }^{3} P_{1}$ and $6 s^{2}{ }^{1} S_{0} \rightarrow 6 s 6 p{ }^{1} P_{1}$ absorptions, respectively.

Smaller $4 f^{13} 5 d^{1} 6 s^{2}$ state anisotropy should also diminish Jahn-Teller splitting of the doublet peaks. Observed splittings for bands (c), (d) and (e) are 25, 9 and 21 $\mathrm{cm}^{-1}$, respectively. Significantly larger values of 80 and $140 \mathrm{~cm}^{-1}$ for bands (a) and (b), respectively, are predicted by the $6 s \rightarrow 6 p$ absorption simulations. Similarity of the pairs of excited- and ground-state interaction potentials in $4 f \rightarrow 5 d$ transitions to those in $6 s \rightarrow 6 p$ transitions implies similar frequencies for the phonons involved. Calculations for the $10 \mathrm{~V}$ site simulated in SecIVB predict 16 and $19 \mathrm{~cm}^{-1}$ for $A_{1}$ and $E$ symmetry phonons, respectively - close to the value of $14 \mathrm{~cm}^{-1}$ deduced from the sideband progression of $A_{1}$ symmetry phonons in Fig. 5 .

\section{Emission Spectrum}

The spectrum resulting from $6 s^{2}{ }^{1} S_{0} \rightarrow 6 s 6 p{ }^{1} P_{1}$ excitation has been discussed in detail in earlier reports [10, 32]. They found corroborating evidence for the presence of a crystal field in the observation of $6 s 6 p{ }^{3} P_{0}$ state decay. The quenching of this state is posited to occur through a Stark-like coupling to the $6 s 6 p{ }^{1} P_{1}$ and $6 s 6 p{ }^{3} P_{1}$ states mediated by a field the order of $\sim 20$ $\mathrm{MV} / \mathrm{m}$ [11]. A common feature of these reports, though, is that they only considered the fluorescence products at energies below those of the excitation energy i.e., in the $<2.6 \times 10^{4} \mathrm{~cm}^{-1}$ range. The present work is the first one to consider the spectrum due to decay from higher energy levels, which are reached through the absorption of a photon by the excited $\mathrm{Yb}\left({ }^{3} P_{0}\right)$ atom. Thus, the emission counterparts to the absorption bands (c), (d) and (e) in Fig. 4 are observed at 29,030 $\mathrm{cm}^{-1}, 37,730 \mathrm{~cm}^{-1}$ and $39,610 \mathrm{~cm}^{-1}$ in Fig. 6 .

The above is important for the proposed single atom detection of $\mathrm{Yb}$ in $\mathrm{sNe} 22$. The imaging of a single 
$n \mathrm{~s}^{2} S$ ground state atom in RG isolation has already been demonstrated for $\mathrm{Ba}$ in solid $\mathrm{Xe}$ through the observation of the $6 \mathrm{~s} 6 \mathrm{p}{ }^{1} P_{1}$ state decay following its resonant excitation [23. But efficient detection of singlet state fluorescence is only possible when its branching ratio to other states is low. Thus, for the majority of $\mathrm{M} / \mathrm{RG}$ systems, in which such fluorescence is heavily quenched by matrix-enhanced intersystem crossing, a proof-of-principle using the $\mathrm{Yb} / \mathrm{Ne}$ system is highly relevant. The latter's ${ }^{3} P_{1}$ state fluorescence has the advantage of being spectroscopically distinct from the source's exciting photons and its decay rate of $\sim 1.47 \mathrm{MHz}$ is orders of magnitude greater than the dark count rate $(\sim$ $25 \mathrm{~Hz}$ ) of widely-available single photon counting modules (e.g. Perkin Elmer AQR-16 SPCM). A drawback, though, of leveraging its triplet manifold for single atom detection is that its relatively long-lived ${ }^{3} P_{0}$ state acts as a population trap into which electrons will be transferred on a timescale that is the order of that state's lifetime $(\sim 17 \mathrm{~s}$ in sNe). Fortunately, the observation here of the $4 f^{13} 5 d^{1} 6 s^{2} \rightarrow 6 s^{2}{ }^{1} S_{0}$ transition, whose decay rate is at least an order of magnitude higher than that of the ${ }^{3} P_{1}$ state, shows that $6 s 6 p{ }^{3} P_{0} \rightarrow 6 p^{2}{ }^{3} P_{1}$ repumping is a viable method for returning the dark state $\mathrm{Yb}$ atom back to the ground state.

For metal atoms isolated in the heavier RGs, structures observed in the absorption spectra due to matrix effects such as multiple site occupation, Jahn-Teller coupling and CFS, usually have counterparts in the emission spectra. In sNe, however, this is rarely the case and emission bands typically have a simple unstructured Gaussian profile [4]. Some matrix effects can still be resolved by lifetime measurements, as each component of the emission band may have distinct decay probabilities. For instance, previous lifetime measurements of the $\mathrm{Yb}\left({ }^{3} P_{0}\right)$ state in sNe were fitted to a triple exponential and the various decay constants attributed to multiple site occupation and isotope composition [10]. However, to identify such components in the lifetimes of all the excited state decays reported here would require a significant upgrade in our apparatus and so is left for future work.

Spin-polarization of ${ }^{171} \mathrm{Yb}$ nuclei in a matrix by optical pumping would enable the kinds of precision experiments envisioned for optically addressable solid-state spin systems. If the hyperfine structure of a particular transition is resolved, then it is expected that the optical pumping efficiency (the fraction of angular momentum transferred from the photon to the atom) will be high. However, if the hyperfine structure is unresolved, the optical pumping efficiency will likely depend on the details of the line-broadening mechanism. The hyperfine structure for the naturally abundant ${ }^{171} \mathrm{Yb}(I=1 / 2,14 \%)$ and ${ }^{173} \mathrm{Yb}(I=5 / 2,16 \%)$ isotopes do not appear to be resolved for any of the transitions studied here. This implies that the optical pumping efficiency maybe low, which could be compensated for by a high number density. Because the transitions involving the inner shell
$4 f$ electrons have unusually narrow features, a more detailed study of them using circularly polarized light has the potential to find evidence of optical pumping.

\section{CONCLUSION}

High-resolution spectroscopy of $\mathrm{Yb}$ atoms in a cryogenic Ne matrix over a wide excitation range revealed five asymmetric absorption features. The two lowest are assigned to outer-shell $6 s \rightarrow 6 p$ transitions to $6 s 6 p^{3} P_{1}$ and ${ }^{1} P_{1}$ atomic states and appear, respectively, as a broad doublet and an asymmetric triplet. Three bands that correspond to inner-shell $4 f \rightarrow 5 d$ transitions to distinct $J=1$ states arising from the $4 f^{13} 5 d^{1} 6 s^{2}$ configuration are much narrower and have rich structural features.

Interpretation of the band structures relied on the modeling of the stable trapping sites of the $\mathrm{Yb}$ atom in a perfect Ne fcc crystal. This indicated that the most energetically stable site is a 10 -atom vacancy of $C_{4 v}$ symmetry and that there are three other stable sites-6-, 8- and 13 -atom vacancies - lying relatively close by in energy. In this environment, any $J=1$ atomic state should split into one-dimensional $A_{1}$ and two-dimensional $E$ representation. The dynamic Jahn-Teller effect will then lift the remaining degeneracy producing the generic $1+2$ or $2+1$ absorption bandshapes observed. The magnitude of the splittings depends on the interaction anisotropy which varies from strong for the ${ }^{1} P_{1}$ state to medium for the ${ }^{3} P_{1}$ state and is largely suppressed for the $4 f^{13} 5 d^{1} 6 s^{2}$ states.

Similarly, the degree of phonon excitation reflects the difference in the ground- and excited-state interaction potentials. It ranges from significant band broadening in the $6 s \rightarrow 6 p$ transitions to the perfectly resolved phonon sideband progression in the $6 s^{2}{ }^{1} S_{0} \rightarrow$ $4 f^{13} 5 d^{1} 6 s^{2}(5 / 2,5 / 2)_{1}$ transition. While multiple site occupation complicates the detailed assignment of all features of the $4 f \rightarrow 5 d$ transitions, the evidence of resolved individual phonon lines for CFS components is very strong as their fits give plausible values for the Huang-Rhys parameter and the lattice displacement.

Following the $\mathrm{Mn} / \mathrm{Kr}$ [19] and Eu/Ar systems [20], the $\mathrm{Yb} / \mathrm{Ne}$ system is only the third example of a metal-rare gas matrix whose crystal field states yield ZPLs with accompanying phonon sidebands. It is the first one for which this occurs on an allowed transition, for which sNe is the matrix host and for which the trapping site has non-polyhedral symmetry. These results also indicate the peculiarities of the semi-quantum Ne matrix. On the one hand, well-defined CFS reflects the classical nature of relatively rigid trapping sites. On the other, the existence of multiple spacious trapping sites of axial symmetry and discernible individual phonon excitations manifest the onset of quantum solid-state physics. 


\section{ACKNOWLEDGMENT}

We would like to thank T. Oka and R. W. Dunford for helpful discussions and the use of their equipment. Experimental work and analysis is supported by Department of Energy, Office of Nuclear Physics, under Contract No. DEAC02-06CH11357, while the simulations are performed in the frame of Russian Science Founda- tion project No. 17-13-01466. H. X. and S. T. P. are supported by the U.S. Department of Energy, Office of Science, Office of Basic Energy Sciences, Division of Chemical Sciences, Geosciences, and Biosciences under contract No. DE-AC02-06CH11357. J. T. S. is supported by Argonne Director's postdoctoral fellowship and R. L. is supported by the China Postdoctoral Science Foundation.
[1] J. H.. Van Vleck. The Dipolar Broadening of Magnetic Resonance Lines in Crystals. Phys. Rev., 74:1168-1183, Nov 1948.

[2] C. Kittel and Elihu Abrahams. Dipolar Broadening of Magnetic Resonance Lines in Magnetically Diluted Crystals. Phys. Rev., 90:238-239, Apr 1953.

[3] M. G. Kozlov and Andrei Derevianko. Proposal for a Sensitive Search for the Electric Dipole Moment of the Electron with Matrix-Isolated Radicals. Phys. Rev. Lett., 97(6), aug 2006.

[4] A. C. Vutha, M. Horbatsch, and E. A. Hessels. Orientation-dependent hyperfine structure of polar molecules in a rare-gas matrix: A scheme for measuring the electron electric dipole moment. Phys. Rev. A, 98:032513, Sep 2018.

[5] Andrew N. Kanagin, Sameer K. Regmi, Pawan Pathak, and Jonathan D. Weinstein. Optical pumping of rubidium atoms frozen in solid argon. Phys. Rev. A, 88:063404, Dec 2013.

[6] Sunil Upadhyay, Ugne Dargyte, David Patterson, and Jonathan D. Weinstein. Ultralong spin-coherence times for rubidium atoms in solid parahydrogen via dynamical decoupling. Phys. Rev. Lett., 125:043601, Jul 2020.

[7] Gary Wolfowicz, F. Joseph Heremans, Christopher P. Anderson, Shun Kanai, Hosung Seo, Adam Gali, Giulia Galli, and David D. Awschalom. Quantum guidelines for solid-state spin defects. Nat. Rev. Mater., pages 1-20, 2021.

[8] W. Weyhmann and F. M. Pipkin. Optical absorption spectra of alkali atoms in rare-gas matrices. Phys. Rev., 137:A490-A496, Jan 1965.

[9] Sunil Upadhyay, Ugne Dargyte, Robert P. Prater, Vsevolod D. Dergachev, Sergey A. Varganov, Timur V. Tscherbul, David Patterson, and Jonathan D. Weinstein. Enhanced spin coherence of rubidium atoms in solid parahydrogen. Phys. Rev. B, 100:024106, Jul 2019.

[10] C.-Y. Xu, S.-M. Hu, J. Singh, K. Bailey, Z.-T. Lu, P. Mueller, T. P. O'Connor, and U. Welp. Optical excitation and decay dynamics of ytterbium atoms embedded in a solid neon matrix. Phys. Rev. Letters, 107(9), aug 2011.

[11] C.-Y. Xu, J. Singh, J. C. Zappala, K. G. Bailey, M. R. Dietrich, J. P. Greene, W. Jiang, N. D. Lemke, Z.-T. Lu, P. Mueller, and T.P. O'Connor. Measurement of the hyperfine quenching rate of the clock transition in Yb171. Phys. Rev. Letters, 113(3), jul 2014.

[12] Vinod Gaire, Chandra S. Raman, and Colin V. Parker. Subnanometer optical linewidth of thulium atoms in raregas crystals. Phys. Rev. A, 99:022505, Feb 2019.

[13] Sunil Upadhyay, Andrew N. Kanagin, Chase Hartzell, Tim Christy, W. Patrick Arnott, Takamasa Momose,
David Patterson, and Jonathan D. Weinstein. Longitudinal Spin Relaxation of Optically Pumped Rubidium Atoms in Solid Parahydrogen. Phys. Rev. Lett., 117:175301, Oct 2016.

[14] T. E. Chupp, P. Fierlinger, M. J. Ramsey-Musolf, and J. T. Singh. Electric dipole moments of atoms, molecules, nuclei, and particles. Rev. Mod. Phys., 91:015001, Jan 2019.

[15] N. N. Kleshchina, I. S. Kalinina, R. Lambo, A. A. Buchachenko, D. S. Bezrukov, and S.-M. Hu. Triplet emission of atomic ytterbium isolated in a xenon matrix. Low Temp. Phys., 45(7):707-714, 2019.

[16] L.-G. Tao, N. N. Kleshchina, R. Lambo, A. A. Buchachenko, X.-G. Zhou, D. S. Bezrukov, and S.-M. Hu. Heat- and light-induced transformations of $\mathrm{Yb}$ trapping sites in an Ar matrix. J. Chem. Phys., 143(17):174306, nov 2015.

[17] C. Crepin-Gilbert and A. Tramer. Photophysics of metal atoms in rare-gas complexes, clusters and matrices. Int. Rev. Phys. Chem., 18(4):485-556, oct 1999.

[18] I. B. Bersuker. The Jahn-Teller Effect. Cambridge University Press, Cambridge, 2006.

[19] O. Byrne, M. A. Collier, M. C. Ryan, and J. G. McCaffrey. Crystal field splitting on $\mathrm{D} \leftrightarrow \mathrm{S}$ transitions of atomic manganese isolated in solid krypton. Low Temp. Phys., 36(5):417-423, may 2010.

[20] Owen Byrne and John G. McCaffrey. Eu/RG absorption and excitation spectroscopy in the solid rare gases: State dependence of crystal field splitting and jahn-teller coupling. J. Chem. Phys., 134(12):124501, mar 2011.

[21] Claudio Cazorla and Jordi Boronat. Simulation and understanding of atomic and molecular quantum crystals. Rev. Mod. Phys., 89(3), aug 2017.

[22] B. Loseth, R. Fang, D. Frisbie, K. Parzuchowski, C. Ugalde, J. Wenzl, and J. T. Singh. Detection of atomic nuclear reaction products via optical imaging. Phys. Rev. C, 99:065805, Jun 2019.

[23] Imaging individual barium atoms in solid xenon for barium tagging in nEXO. Nature, 569(7755):203-207, apr 2019.

[24] J-F Wyart and P Camus. Extended Analysis of the Emission Spectrum of Neutral Ytterbium (Yb I). Phys. Scr., 20(1):43-59, jul 1979.

[25] R Zinkstok, E J van Duijn, S Witte, and W Hogervorst. Hyperfine structure and isotope shift of transitions in yb i using UV and deep-UV cw laser light and the angular distribution of fluorescence radiation. J. Phys. B, 35(12):2693-2701, jun 2002.

[26] B. Karaçoban and L. Özdemır. Energies, Landé Factors, and Lifetimes for Some Excited Levels of Neutral Ytter- 
bium $(\mathrm{Z}=70)$. Acta Phys. Pol. A, 119(3):342-353, mar 2011.

[27] Sefik Suzer and Lester Andrews. Optical spectra of yb atoms and dimers in rare gas matrices. J. Chem. Phys., 89(9):5514-5516, nov 1988.

[28] Guokui Liu. Advances in the theoretical understanding of photon upconversion in rare-earth activated nanophosphors. Chem. Soc. Rev., 44(6):1635-1652, 2015.

[29] Yong Zhang. Applications of Huang-Rhys theory in semiconductor optical spectroscopy. J. Semicond., 40(9):091102, sep 2019.

[30] Max Wagner. Structural form of vibronic bands in crystals. J. Chem. Phys., 41(12):3939-3943, dec 1964.

[31] Guokui Liu. A degenerate model of vibronic transitions for analyzing 4f-5d spectra. J. Lumin., 152:7-10, aug 2014.

[32] R. Lambo, A. A. Buchachenko, L. Wu, Y. Tan, J. Wang, Y. R. Sun, A.-W. Liu, and S.-M. Hu. Electronic spectroscopy of ytterbium in a neon matrix. J. Chem. Phys., 137(20):204315, nov 2012.

[33] Nadezhda N. Kleshchina, Inna S. Kalinina, Iosif V. Leibin, Dmitry S. Bezrukov, and Alexei A. Buchachenko. Stable axially symmetric atomic impurity in an fcc solid-ba in rare gases. J. Chem. Phys., 151(12):121104, sep 2019.

[34] Ronald A. Aziz and M.J. Slaman. The ne-ne interatomic potential revisited. Chemical Physics, 130(1-3):187-194, feb 1989.

[35] Qiang Zhu, Li Li, Artem R. Oganov, and Philip B. Allen. Evolutionary method for predicting surface reconstructions with variable stoichiometry. Phys. Rev. B, 87(19), may 2013.

[36] Barry Davis and John G McCaffrey. Luminescence of Atomic Barium in Rare Gas Matrices: A TwoDimensional Excitation/Emission Spectroscopy Study. J. Phys. Chem. A, 122(37):7339-7350, 2018.

[37] Barry M. Davis, Benoit Gervais, and John G. McCaffrey. An investigation of the sites occupied by atomic barium in solid xenon-A 2D-EE luminescence spectroscopy and molecular dynamics study. J. Chem. Phys.,
148(12):124308, 2018.

[38] Nadezhda N. Kleshchina, Inna S. Kalinina, Iosif V. Leibin, Dmitry S. Bezrukov, and Alexei A. Buchachenko. Stable axially symmetric atomic impurity in an fcc solid-Ba in rare gases. J. Chem. Phys., 151(12):121104, sep 2019.

[39] Barry M. Davis and John G. McCaffrey. Aborption spectroscopy of heavy alkaline earth metals $\mathrm{Ba}$ and $\mathrm{Sr}$ in rare gas matrices - CCSD $(\mathrm{T})$ calculations and atomic site occupanciesancies. J. Chem. Phys., 144(4):044308, 2016.

[40] Maryanne Ryan, Martin Collier, Patrick de Pujo, Claudine Crépin, and John G. McCaffrey. Investigations of the optical spectroscopy of atomic sodium isolated in solid argon and krypton: Experiments and simulations. J. Phys. Chem. A, 114(9):3011-3024, sep 2009.

[41] Jerry A. Boatz and Mario E. Fajardo. Monte Carlo simulations of the structures and optical absorption spectra of $\mathrm{Na}$ atoms in Ar clusters, surfaces, and solids. J. Chem. Phys., 101(5):3472-3487, sep 1994.

[42] J. P. Visticot, P. de Pujo, J. M. Mestdagh, A. Lallement, J. Berlande, O. Sublemontier, P. Meynadier, and J. Cuvellier. Experiment versus molecular dynamics simulation: Spectroscopy of $\mathrm{Ba}-(\mathrm{Ar}) \mathrm{n}$ clusters. J. Chem. Phys., 100(1):158-164, jan 1994.

[43] A. I. Krylov, R. B. Gerber, and R. D. Coalson. Nonadiabatic dynamics and electronic energy relaxation of $\operatorname{cl}(2 \mathrm{p})$ atoms in solid Ar. J. Chem. Phys., 105(11):4626-4635, sep 1996.

[44] Cindy I. Hancox, S. Charles Doret, Matthew T. Hummon, Linjiao Luo, and John M. Doyle. Magnetic trapping of rare-earth atoms at millikelvin temperatures. Nature, 431(7006):281-284, sep 2004.

[45] R. V. Krems and A. A. Buchachenko. Electronic interaction anisotropy between open-shell lanthanide atoms and helium from cold collision experiment. J. Chem. Phys., 123(10):101101, sep 2005.

[46] Brendan Healy, Paul Kerins, and John G. McCaffrey. Metal atom ( $\mathrm{Zn}, \mathrm{Cd}$ and $\mathrm{Mg}$ ) luminescence in solid neon. Low Temp. Phys., 38(8):679-687, aug 2012. 